Eugenio Coseriu (Tübingen)

\title{
Sprach- und Textstrukturen
}

\author{
Transkription von Marius Nagy ${ }^{1}$
}

Meine sehr verehrten Herren Kollegen, meine Damen und Herren,

ich möchte zunächst mich sehr herzlich dafür bedanken bei dem Studium generale, daß mir die Gelegenheit geboten wird, mit Ihnen und vor Ihnen über den Begriff Struktur aus der Sicht der Sprachwissenschaft zu sprechen.

Zuerst etwas zur Struktur dieses Vortrags. Der Vortrag besteht aus drei Teilen. Im ersten Teil werde ich versuchen, den Begriff Struktur im Allgemeinen, wenn auch vor allem mit und auf Grund von sprachwissenschaftlichen Beispielen zu klären. Im zweiten Teil werde ich dann die Anwendung dieses Begriffes auf das Gebiet der Sprachwissenschaft behandeln. Im dritten Teil werde ich mich auf Leistung und Grenzen der strukturalistischen Sprachauffassung und der strukturalistischen Methode beziehen und werde versuchen zu zeigen, wie der Strukturalismus überwunden werden kann und in gewisser Hinsicht auch muß.

1. Zum ersten Teil: der Begriff Struktur. Es gibt eine lockere Verwendung und eine strenge Verwendung des Begriffes Struktur. Was beiden Verwendungen gemeinsam ist, ist der sogenannte Antiatomismus, d.h. die Tatsache, daß die Fakten nicht in ihrer jeweiligen Individualität als Einzelfakten betrachtet werden, sondern als Relationen zueinander, d.h. unter Struktur versteht man zuerst und allgemein Form der Relationen zwischen Fakten auf irgendeinem Gebiet. Da aber Relationen, die man jeweils in Betracht zieht, verschiedenartig sein können, ist auch der Inhalt des Begriffes, selbst der stoffliche Inhalt des Begriffes selbst sehr oft verschieden.

1.1. Was hingegen die beiden Verwendungen des Begriffes und des Wortes Struktur voneinander unterscheidet, ist folgendes: Die lockere Verwendung bleibt bei dem allgemeinen Sinn des Strukturbegriffes, d.h. Struktur von irgendwelchen Relationen, die auch unendlich sein können und die heterogen sein können. Man hat in der Sprachwissenschaft bisweilen gerade diesen Begriff Struktur verwendet, in der Strukturellen Lexikologie von Matoré insbesondere und in ihrer Fortsetzung durch Pierre Guiraud. In diesem Fall handelt es sich um ein Zentrum, als Zentrum gilt ein Wort, und es werden dann die verschiedenen möglichen Relationen dieses Wortes zu anderen Wörtern im Wortschatz einer Sprache festgestellt, sowohl die materiellen Ähnlichkeiten als auch zum Beispiel die metaphorischen

1 Dieser unveröffentlichte Text, der im Archiv Coseriu unter den Kennziffer B XXII, 54 zu finden ist, ist die Transkription eines von Eugenio Coseriu an der Universität Mainz im Sommersemester 1979 gehaltenen Vortrags (des Eröffnungsvortrags einer Reihe des Studium generale in Mainz zum Thema Struktur). Der Text wurde an der Universität Mainz auf der Basis einer Tonbandaufnahme transkribiert; das Typoskript enthält einige Korrekturen, die nicht von der hand Coserius stammen, siehe hier). Wir haben die in der Transkription enthaltenen Lücken wiederhergestellt, einige Inkohärenzen korrigiert und ein paar Anmerkungen angefügt. Um die Besonderheiten der coseriuschen Prosa zu respektieren, haben wir die Absätze nummeriert. 
Relationen als auch die Relationen über die Sachen, die bezeichnet werden usw. Dies hat gerade Matoré mit dem Wort ,art“ im Französischen getan, wo er festgestellt hat, mit welchen anderen Wörtern ,,art“ in irgendeinem Zusammenhang zu einer bestimmten Zeit stand, und er ist natürlich dann zu ,,artiste“ gekommen, zu „,marchand“, zu ,peintre“, zu „goût“ usw. also einer ganzen Menge von Relationen, die an sich heterogen sind und die man dann jeweils fortsetzen kann; denn wenn man z.B. bis „marchand“ gekommen ist, dann kann man zu „marché“ usw. kommen und dann zu „,vendre“ und zu ,acheter“ usw ${ }^{2}$. Bei Guiraud geht es im Grunde um das Gleiche, jedoch noch mit einer Erweiterung. Die Erweiterung besteht darin, daß er auch die rein materiellen Assoziationen feststellt d.h. die Assoziationen durch materielle Ähnlichkeit, so etwa in den Zeichnungen von Guiraud über die Katzen. [Vielleicht werden Sie diese] Zeichnungen kennen, wo dann das mot „chat“ in Zusammenhang gebracht wird mit „,charrue“, „,château“, „,chanoine“, d.h. mit all den Wörtern die etwas mit „,chat“ zu tun haben und die sozusagen das Wort „chat“ zu enthalten scheinen ${ }^{3}$. Diese Art Struktur wollen wir besser Konfiguration nennen, um sie gerade von der strengen Verwendung des Wortes Struktur und des Begriffes Struktur zu unterscheiden ${ }^{4}$. Diese Konfiguration ist einerseits heterogen andererseits ist sie grundsätzlich unendlich, d.h. man hat keinen Grund an einem bestimmten Punkt halt zu machen. Guiraud hat das Wort „,chat“ mit ungefähr 2200 anderen Wörtern in Verbindung gesetzt; er hätte das auch noch weiter machen können, denn von jedem anderen Zentrum kann man weitere Assoziationen feststellen.

1.2. Was den strengen Gebrauch des Wortes betrifft, so ist für diesen Gebrauch folgendes charakteristisch. Es geht erstens um homogene Relationen, d.h. Relationen gleicher Art, z.B. wenn materielle, dann gerade nur die materiellen, wenn inhaltliche nur inhaltliche, wenn formale [z.B. was Rhythmus betrifft], dann gerade nur diese Relationen. Zweitens geht es um innere Relationen, innerhalb eines geschlossenen, eines abgeschlossenen Gebiets. Das Gebiet gilt als Gegenstand und dieser Gegenstand hat eine Struktur in der Hinsicht, daß er - der Gegenstand - eine innere Beschaffenheit aufweist, daß seine Teile oder die Fakten, die dieses Gebiet enthält, in bestimmten Relationen zu einander stehen. Dies ist auch der Begriff Struktur, der in der Sprachwissenschaft gebraucht wird, und zwar mit diesem Namen erst in unserem Jahrhundert allgemein. Vereinzelt hat man immer wieder von Struktur der Sprache gesprochen, aber noch früher von dem Begriff, nicht aber mit diesem Namen. Ich möchte darauf hinweisen in diesem Zusammenhang, daß der Begriff Struktur dann überhaupt nichts Geheimnisvolles an sich hat, wenn man ihn so nennt, wie man ihn früher nannte: früher hat man von Bau gesprochen. Das heißt, es geht darum, wie ein Gegenstand gebaut ist, um die Bauweise, wie er gemacht ist, welches seine innere Beschaffenheit ist. Humboldt gebraucht fast regelmäßig gerade dieses Wort, diesen Terminus „Bau“ für das, was heute Struktur der Sprache genannt wird. Manchmal gebraucht er auch Typus und manchmal gebraucht er Form, wenn auch mit verschiedenen Schattierungen ${ }^{5}$. In dieser Hinsicht geht es also um die inneren

Georges Matoré, 1953, La méthode en lexicologie. Domaine français, Paris, Didier, p. 99-117

Pierre Guiraud, 1956, Les champs morphosémantiques, Bulletin de la Société Linguistique de Paris, 56, S.

265-288 ; Pierre Guiraud, 1966, Le champ morphosémantique des noms du chat, Bulletin de la Société Linguistique de Paris, 61, S. 128-145

4 Über die assoziativen Konfigurationen, Vgl. Eugenio Coseriu/Horst Geckeler, 1981, Trends in Structural Semantics, Gunter Narr Verlag, Tübingen, S. 16-17

5 Wilhelm von Humboldt, 1903-36, Gesammelte Schriften, hrsg. von der Preußischen Akademie der Wissenschaften, Band VII, S. 165f.; Vgl. auch Eugenio Coseriu, 2002, Wilhelm von Humboldt und die Sprachtypologie, in K. Ezawa und al., (Hrsg.), Linguistik jenseits des Strukturalismus, Gunter Narr Verlag, Tübingen, S. 36; Jürgen Trabant, 1986, Apeliotes oder der Sinn der Sprache. Wilhelm von Humboldts Sprach-Bild, Fink, München, S. 173 
Relationen und in dieser Hinsicht unterscheidet sich der Begriff Struktur vom Begriff Form. Die Form in diesem Falle ist dann die äußere Form eines Gegenstandes. Die Struktur ist die innere Beschaffenheit des Gegenstandes oder des Gebietes, das man als abgeschlossenes Gebiet untersucht. Es gibt trotzdem auch bei dieser strengen Verwendung des Begriffes Struktur noch die Möglichkeit, daß die Ordnung, die man feststellt, oder die man bei den Fakten einführen will, entweder eine willkürliche und eine konventionelle oder eine objektive und reale Ordnung ist. Willkürlich ist eine Ordnung, wenn sie mit subjektiven Kriterien aufgestellt wird. Man könnte zum Beispiel die Wörter einer Sprache ohne weiteres aufgrund des Kriteriums, ob sie mir als Wörter gefallen oder nicht gefallen, ordnen. Das wäre eine willkürliche Ordnung, wenn auch sicherlich eine motivierte - denn ich könnte meine Gründe dafür haben. Eine Ordnung ist hingegen objektiv, wenn sie aufgrund von objektiven Kriterien erfolgt. Eine objektive Ordnung kann aber ihrerseits konventionell sein oder real sein. Sie ist konventionell, wenn sie nicht der tatsächlichen Ordnung die durch den Sinn selbst des Gegenstandes eben gegeben ist, entspricht. Wenn ich zum Beispiel die Wörter einer Sprache nach ihrer Länge ordne, so ist das eine objektive Ordnung, denn das Kriterium ist objektiv, jedoch ist das keine reale Ordnung, es entspricht eigentlich nicht den tatsächlichen Relationen, die für Sprache maßgebend sind. Oder die alphabetische Reihenfolge, die wir für bestimmte praktische Zwecke in den Wörterbüchern benutzen. Das ist sicherlich eine objektive Ordnung, sie hat aber mit einer realen Ordnung, mit der realen Struktur der Sprache kaum etwas zu tun. Ich sage kaum etwas zu tun, denn es wäre auch möglich z.B. in diesen beiden Fällen, daß gerade auch die Länge einen Sinn in einer bestimmten Sprache hat. Wenn z.B. alle kurzen Wörter Substantive wären in einer bestimmten Sprache oder wenn alle längeren Wörter überall in dieser Sprache Ableitungen wären, dann würde diese Ordnung einer auch objektiven, einer realen Ordnung entsprechen. Sogar die materielle Beschaffenheit, die phonische Beschaffenheit der Wörter könnte einen realen Sinn haben. Sagen wir z.B. ein Wort, das auf „d“ auslautet im Lateinischen, ein solches Wort Kann im Lateinischen nur Pronomen sein oder ein Adverb oder eine Präposition. Es kann nichts anderes sein, nicht Substantiv z.B. oder Verb, oder Adjektiv. Oder wenn ein solches Wort als Substantiv in einem lateinischen Text erscheint, dann ist ein solches Wort gerade kein lateinisches Wort. Man erkennt es gerade als nicht-lateinisch, weil es dies aufweist; wenn man z.B. David, oder Galzad oder solches in einem lateinischen Text findet. Sonst hat man im Lateinischen nur $a d$, apud, quid, haud. Diese Fakten könnten eventuell auch einer realen Ordnung entsprechen, das ist aber meist gerade nicht der Fall.

1.3. In der Sprache bedeutet eine reale Ordnung eine solche, die der tatsächlichen Struktur der Sprache entspricht, eine Ordnung, die funktionell in der Sprache ist. Die Sprache ist im ganzen Funktion, die Sprache funktioniert und funktioniert zum Ausdruck von Inhalten. Dadurch ergibt sich auch das allgemeine reale Kriterium für die Struktur der Sprache, nämlich das Funktionieren zum Ausdruck von Inhalten. Dies ist auch das Kriterium in der strukturellen Sprachwissenschaft, d.h. was ist in einer Sprache funktionell. Und funktionell in einer Sprache bedeutet stets funktionell in Bezug auf einen Inhalt in dieser Sprache, entweder so, daß es um die Relationen geht, die den Inhalt unmittelbar betreffen, oder so, daß es um die Relationen geht, die das Materielle, den Ausdruck eines Inhalts, betreffen. Dies ist anfangs leicht gesagt, und es war auch die Intuition im Strukturalismus. Was man anfangs, zum Teil auch bis heute, im Strukturalismus nicht gesehen hat, war, daß dies schon einen Ort der Sprache in einer Gesamtstruktur der Sprache überhaupt voraussetzt, d.h. wenn wir tatsächlich den Inhalt als das Kriterium annehmen, dann müssen wir zugleich feststellen, daß wir in der 
Sprache überhaupt zunächst eine Struktur gerade der Sprache überhaupt oder der Sprache im allgemeinen feststellen müssen, nämlich folgende Struktur: 1. zuerst das Sprechen im allgemeinen, eine Ebene, die das ganze Sprechen in allen Sprachen betrifft; 2. die Sprachen oder Einzelsprachen und 3. die Texte, d.h. die universelle Ebene, die historische Ebene und die individuelle Eben, diese letztere des Redeaktes oder der zusammenhängenden Reihenfolge von Redeakten, das, was wir Text nennen können. In andere Sprachen haben wir dafür bessere Termini; im Französischen z.B. kann man „discours“ sagen, so daß man „discours“ und ,texte“ noch als die Handlung und ihr Ergebnis unterscheiden kann ${ }^{6}$.

1.4. Diese Ebenen sind deshalb zu unterscheiden, weil sie autonom sind, gerade durch den Inhalt, den sie jeweils ausdrücken. Das, was zum Sprechen im allgemeinen gehört, ist ein Inhalt, den wir Bezeichnung nennen dürfen, das heißt, es geht darum, daß sich all das Sprachliche auf Außersprachliches bezieht; das Sprachliche etwas in der außersprachlichen Welt benennt, gleichgültig, ob diese Welt jetzt die physische Welt oder eine imaginäre Welt ist, ja, gleichgültig auch, ob diese Welt die Sprache selbst als Teil der Welt ist; das heißt die Sprache gehört selbst zur Welt und kann als solche durch die Sprache wiederum genannt, benannt werden. Dieses Verhältnis, gerade zum Außersprachlichen, ist das was alle Sprachen und alle Sprechen charakterisiert, und die Möglichkeit der Bezeichnung ist grundsätzlich die gleiche als Möglichkeit in allen Sprachen. D.h. grundsätzlich kann man alles durch jede Sprache ausdrücken, alles durch jede Sprache benennen, und auch das natürlich, was bisher aus empirischen Gründen in einer bestimmten Sprachgemeinschaft noch nicht benannt worden ist, zum Beispiel weil die entsprechende Klasse von Gegenständen in dieser Gemeinschaft unbekannt ist. Die Möglichkeit ist aber durch jede Bezeichnung gegeben, und alle Sprachen fallen gerade in der Bezeichnung miteinander zusammen.

1.5. Die Art des Inhalts, die man in den Sprachen, in den Einzelsprachen findet, kann man Bedeutung nennen. Das ist eine besondere Gestaltung der Möglichkeiten der Bezeichnung in einer bestimmten Sprache. Sagen wir zum Beispiel, diese Tatsache, daß man sich mit einem anderen Gegenstand bewegt, diese Tatsache kann durch verschiedene Wörter in verschiedenen Sprachen, und durch verschiedene Bedeutungen bezeichnet werden. Die Einteilung, das, was eigentlich die Bedeutung in der Sprachen ausmacht, ist jedoch sehr oft und ist grundsätzlich sogar verschieden. Im Deutschen unterscheidet man „,mit Richtung“ und „ohne Richtung“, „,bringen“ und „tragen“; im Italienischen macht man normalweise überhaupt keinen Unterschied, in jedem Fall „portare“. Im Spanischen und im Portugiesischen macht man einen Unterschied je nach der Richtung und zwar hinsichtlich der Personen „traer“ = ,in Richtung auf den Ort der ersten Person“ und „llevar“ = ,in Richtung auf der Ort der zweiten oder der dritten Person“. Im Französischen macht man wiederum einem anderen Unterschied, nämlich, ob das, was sich sekundär bewegt, sich selbsttätig bewegt oder nicht. Wenn selbsttätig, dann heißt das „mener“, wenn zum Beispiel ein Lebewesensich mit einem anderen zusammen bewegt. Wenn nicht selbsttätig, dann heißt es das ,porter“, daß es sich nicht selbsttätig bewegt. Ich kann in dieser Hinsicht, wie ich in den Vorlesungen sage, auch in Frankreich meine fiancée zum Beispiel ins Kino ,porter“; aber in diesem Fall trage ich sie. Im Italienischen hingegen sagen wir ,,portare“ ohne weiteres, weil das Italienische diesen Unterschied überhaupt nicht kennt. Im Deutschen könnte ein junger

$6 \quad$ Vgl. Eugenio Coseriu, 1988, Sprachkompetenz. Grundzüge der Theorie des Sprechens, hrsg. von Heinrich Weber, Francke Verlag, Tübingen, S. 75ff. ; Eugenio Coseriu, 1994, Textlinguistik. Eine Einführung, hrsg. von Jörn Albrecht, Francke Verlag, Tübingen S. 10 
Mann seiner Verlobten oder Geliebten sagen „du schmeckst mir“, aber dann würde er tatsächlich meinen, daß sie ihm so wie eine Speise schmeckt. Im Italienischen, wo man den Unterschied zwischen „schmecken“ und „gefallen“ nicht hat, sagt man ohne weiteres „piacere“ - „tu mi piaci“, und wenn man das sagen wollte, was man im Deutschen mit „du schmeckst mir“ sagt, dann müsste man sagen „,sei saporita“ oder „,vorrei mangiarti“ — ,ich möchte dich aufessen“. Also diese Einteilung der Möglichkeiten der Bezeichnung ist die Bedeutung.

1.6. In den Texten hat man eine andere Art von Inhalt, die gerade nur in den Texten vorkommt, und die ich aufgrund einer langen Tradition Sinn nenne, so wie sie sogar in der Gemeinsprache oft genannt wird, und so, wie sie schon in der Antike genannt wurde, vor allem in der Theorie der Übersetzung. Unter Sinn verstehe ich das, was mit der Bedeutung und mit der Bezeichnung in den Texten tatsächlich gesagt wird; das, was über die Bedeutung und über die Bezeichnung hinaus durch Situation, Kontext und Kenntnis der Sachen usw. determiniert wird ${ }^{7}$. Man hat zum Beispiel bemerkt, daß die Worte ,es ist kalt“ von einer Dame gesagt, verschiedene Bedeutungen haben könnten. Im Text, auf den ich mich beziehe, wird gesagt, ja diese Worte könnten bedeuten zum Beispiel „du sollst das Fenster zumachen“, oder sie können bedeuten „du solltest die Heizung höher stellen“, oder sie könnten auch bedeuten „es ist draußen oder hier folgende Temperatur“; sie könnten auch bedeuten „du darfst ruhig näher-rücken“ usw. Es handelt sich hier um eine Verwechslung. „Es ist kalt“ bedeutet nichts anderes im Deutschen, als „es ist kalt“. Das heißt „es ist kalt“ kann nicht etwa „du darfst näher-rücken“ bedeuten. Wenn ,es ist kalt" in einer anderen Situation gebraucht wird, dann kann man dies überhaupt nicht verstehen, und wenn man gerade diese Dame und ihre Gewohnheiten nicht kennt, dann kann man zu dieser Bedeutung mittels der deutschen Sprache nie kommen. Das, was in der deutschen Sprache mit der deutschen Sprache gesagt ist, ist etwas das, was man auf Italienisch „fa freddo“ oder auch auf Französisch ,il fait froid“ nennt. Was hingegen stimmt, ist, daß diese sogenannten Bedeutungen den Sinn dieses kleinen Textes ausmachen können. Das heißt, es geht hier nicht um die Bedeutung, sondern um den Sinn des Textes. Die Bedeutung wird verwendet, um einen Sinn zu vermitteln. In semiotischer Hinsicht müsste man sagen, daß so wie das Materielle, das signifiant, ein signifié, eine Bedeutung hat, hier im Text Bedeutung und Bezeichnung zusammen zu einem „signifiant“" werden für einen anderen Inhalt, für den Sinn; das heißt, all das, was tatsächlich gesagt wird, und all das, was bezeichnet wird in einem Text, meint seinerseits etwas: damit ist etwas gemeint, und das Gemeinte ist der Sinn. In dieser Hinsicht sage ich, Tolstoi spricht nicht von Anna Karenina, er spricht mittels Anna Karenina; das ist auch ein Ausdrucksmittel, und das, was er von Anna Karenina sagt, und das, was mit Anna Karenina im Roman geschieht, das ist nur wiederum ein signifiant, wiederum ein Zeichen für einen Sinn und man kann sich vernünftig fragen, was hat eigentlich Tolstoi mit Anna Karenina gemeint. So wie man sich tatsächlich und vernünftig fragen kann, was Kafka mit Gregor Samsa gemeint hat. Denn Gregor Samsa ist ein Ausdrucksinstrument und das, was mit Gregor Samsa geschieht, ist nicht der Sinn, sondern gerade die materielle Seite des Textzeichens für einen Sinn, den man ermitteln muss und den man auch gerade zum Teil strukturell ermitteln kann. In dieser Hinsicht also drei verschiedene Ebene in der Sprache.

7 Eugenio Coseriu, 1994, op.cit., S. 64f. Vgl. auch Jürgen Trabant, 1975, Vom Sinn, in B. Schlieben-Lange (Hrsg.), Sprachtheorie, Hamburg, S. 277-285 
2. Der Strukturalismus hat sich auf die Ebene der Einzelsprachen konzentriert und hat den Bau gerade, die Art wie die Sprachen gemacht sind, wie jede Sprache gemacht ist, festgestellt. $\mathrm{Zu}$ jeder Sprache insbesondere. Es sind also die einzelsprachlichen Strukturen, die bisher vor allem untersucht worden sind. Nicht die Strukturen des Sprechens im Allgemeinen, weil diese Relationen überhaupt keine Struktur haben. Sie haben jeweils nur eine Form, nur sprachlich und außersprachlich. Aber auch nicht die Strukturen der Texte, die sicherlich auch sprachliche Strukturen sind. Was heißt Strukturen in den Einzelsprachen feststellen? Es heißt so viel wie die Einheiten, die in einer Sprache tatsächlich funktionieren, feststellen und die Relationen der verschiedenen Einheiten jeweils homogen zueinander feststellen, d.h. die Form der Relationen feststellen, die Art wie die Sprache gebaut ist. Das Kriterium der Funktion und der Funktion schlechthin, die Funktion $\kappa \alpha \tau^{\prime} \dot{\varepsilon} \xi o \chi \eta ́ v$ auf dieser Ebene wird gerade die Bedeutung, d.h. die bestimmte Einteilung der Möglichkeiten der Bezeichnung in einer bestimmten Sprache. Es ist möglich, auch in Bezug auf die Sprache konventionelle, wenn auch objektive Strukturen festzustellen. So wie man zwar objektive Strukturen feststellen kann, sagen wir daß das ein Haus mit verschiedenen Stockwerken ist, so könnte man tatsächlich die Struktur des Hauses feststellen, das heißt, diese Einteilung nach Stockwerken und innerhalb der Stockwerke dann die Räume. Aber man könnte auch sagen, ja, das Haus ist folgendermaßen gebaut und dass dann die verschiedenen Teile so ineinandergreifen, das heißt, man hätte hier solche Strukturen, die zwar objektiv, aber konventionell wären. Dies wird bisweilen auch in der Sprachwissenschaft getan, vor allem in der Syntax, wo z.B. Strukturen festgestellt werden, wie Subjekt - Verb zu jedem Satz, wobei dies überhaupt nicht die reale sprachliche Struktur ist. Und dann spricht man von Ergänzungen des Verbs oder Determinationen des Verbs und Ergänzungen des Subjekts oder Determination des Subjekts. In Wirklichkeit hat man, und zwar in allen Sprachen, zunächst einmal etwas, was den Satz konstituiert, dann die erste Referenz des Verbs ein Subjekt, das überhaupt nicht das sein muss, was bei uns Subjekt ist, und dann zwar Subjekt mit seinen Determinationen, aber nie Verb mit seinen Determinationen; denn all die Ergänzungen, die man „compléments du verbe“ nennt, ergänzen eigentlich nicht das Verb, sondern den Ganzen Nukleus des Satzes. Man kann in der Tat nicht nach einer Ergänzung mit dem Verb allein fragen, z.B. „wo stand“, „wo war“ usw., sondern nur mit dem Nukleus. Ein Subjekt muss schon mitgegeben werden. Die eigentlichen Determinationen des Verbs sind die sogenannten Modalverben. Diese modifizieren tatsächlich nur das Verb allein. Dies ist aber eine Einzelheit, nur um zu zeigen, daß man zwar auch in der Sprachwissenschaft ohne weiteres von Strukturen sprechen kann, die in praktischer Hinsicht wichtig sein können, damit macht man eine ganze Grammatik z.B. und man glaubt sogar, daß das auch eine Theorie der Grammatik und der Sprache überhaupt ist. Dies ist aber keine reale Struktur, sondern eine konventionelle.

2.1. Eine reale Struktur stellt man fest, wenn man dem impliziten Prinzipien der strukturellen Sprachbetrachtung folgt, und diese impliziten Prinzipien sind leider nie ausdrücklich formuliert worden, sie sind aber fast überall bei den Strukturalisten implizit gemeint ${ }^{8}$.

2.1.1. Diese Prinzipien sind erstens - und das ist das determinierende Prinzip - die Funktionalität. Es existiert als Einheit in einer Sprache das, was in dieser Sprache funktionell ist. Das was in dieser Sprache funktionell abgegrenzt werden kann, und funktionell abgrenzen

8 Über die "Prinzipien des analytischen Strukturalismus", Vgl. Eugenio Coseriu, 1981, Lecciones de lingüística general, Mardid, Gredos, Kap. VII-VIII, und Jörn Albrecht, 2000, Europäischer Strukturalismus. Eine forschungsgeschichtlicher Überblick, Tübingen und Basel, A. Francke Verlag, S. 57 
bedeutet auf der Ausdrucksebene eine Einheit, wenn dieser Einheit auch eine Einheit auf der Inhaltsebene entspricht; und auf der Inhaltsebene eine Einheit, wenn dieser Einheit auch ein besonderer Ausdruck entspricht. Man kann ohne weiteres auch im Deutschen „singen“ einmalig semelfaktiv und „singen“ mehrmalig frequentativ gebrauchen, man hat aber dafür keine zwei Formen, man sagt in beiden Fällen „,singen“, und man versteht, daß das „einmalig“ ist oder daß das „,das übliche“ ist, durch Kontext und Situation. Die deutsche Sprache sagt das nicht, deshalb hat das Deutsche auch nur eine Einheit „singen“. Eine bestimmte Form des Lateinischen hingegen hatte „canere“ und „cantare“, zwei Ausdrücke. Das eine für das einmalige, für das Semelfaktive, das andere für das Frequentative; also: ,puer canit“ heißt „Der Knabe singt“ - man hört ihn jetzt - dagegen ,puer cantat“, ,,der Knabe singt“, vielleicht in diesem Augenblick nicht, aber doch immer wieder. Man hat hier zwei Inhalte, weil man auch zwei Formen hat. Auch in der deutschen Sprachgemeinschaft kann man sicherlich die Art, wie eine Person alt ist, von der Art, wie eine Pflanze alt ist, und von der Art, wie eine Sache alt ist, unterschieden, und man könnte sagen, ja, ,alt" hat jeweils eine andere Bedeutung. Keineswegs, weil das Deutsche dies gerade nicht unterschiedet, wohl aber das Lateinische, weil das Lateinische gerade dafür „senex“ einmal für Personen, „vetulus“ für Pflanzen und Tiere, und „,vetus“ für Sachen hatte. D.h. es existiert in einer Sprache als Einheit das, was in dieser Sprache als Einheit funktioniert, was funktionell abgegrenzt werden kann.

2.1.2. Das zweite Prinzip ist das Prinzip der Opposition. Es betrifft das Wie des Funktionierens. Die Einheiten funktionieren dadurch, daß sie zum Teil einander gleich und zum Teil verschieden sind; d.h. „senex“ und „vetus“ z.b. haben einen Teil, der bei beiden Einheiten gleich ist - es ist nämlich hier das hohe Alter - und etwas, was diese Einheiten voneinander unterscheidet, das ist „für Personen“, das ist „für Sachen“ und diese zusammen funktionieren wieder in Opposition, z.B. zu ,,iuvenis“ und zu „novus“, die mit diesen die Tatsache zusammen haben, daß es um das Alter, um die Dauer des Lebens geht. Hier aber gerade das Gegenteil des hohen Alters. Durch die Opposition ergibt sich gerade bei der strukturalistischen Sprachbetrachtung die Möglichkeit, auch verschiedene andere unterscheidende Züge festzustellen, so daß eine Einheit dann als Summe von diesen unterscheidenden Zügen aufgefasst werden kann. In dieser Hinsicht sagte schon Saussure auf eine Weise, die sicherlich paradox war, in der Sprache gebe es nur Differenzen, nur Unterschiede. Natürlich sind die Unterschiede alle gerade positiv. Aber die Unterschiede, diese unterscheidenden Züge sind diejenigen, die die Einheiten in analytischer Hinsicht ausmachen. Das ist der Grundbegriff des Strukturalismus, diese Möglichkeit, die Einheiten selbst zu analysieren, in den Einheiten eine Struktur festzustellen durch die Relationen zu anderen Einheiten.

2.1.3. Das dritte Prinzip ist das Prinzip des Systems. Es wäre besser, wenn das im Deutschen zulässig wäre zu sagen: der Systematizität, ein Prinzip, das eine Erwartung betrifft, die Tatsache nämlich, daß man damit rechnen kann, daß die gleichen Oppositionen sich in einer Sprache wiederholen, daß sie mehrmals erscheinen. Es ist überhaupt nicht notwendig - und in dieser Hinsicht wurde der Strukturalismus falsch interpretiert -, daß die Oppositionen sich stets wiederholen, wo sie sich wiederholen könnten. Es ist aber eine Tatsache - und deshalb kann man dies erwarten -, daß die Oppositionen mehrmals vorkommen. D.h. die Opposition, die man z.B. bei „traer“/,llevar“" hat, hat man im Spanischen noch einmal bei „venir“/,,ir“; auch so: „,in Richtung auf den Ort der ersten Person allein“, „,in Richtung auf den Ort der zweiten und dritten Person“. Das, was man im Deutschen einmal hat, bei „stehen“, „sitzen“, 
„liegen“, hat man noch einmal bei den nichtstatischen, sondern dynamischen Verben „stellen“, „setzen“, „legen“, die wiederum in die Positionen weisen, die gerade durch „stehen“, „sitzen“, „liegen“ benannt sind. Das, was man einmal z.B. in der Phonologie bei d:t hat in einer Sprache, hat man dann in der gleichen Sprache noch einmal bei b: $p$, noch einmal bei g:k, d.h. jeweils die gleiche Opposition. Ob sich die Opposition wirklich wiederholt überall, das ist eine Tatsache, die jeweils festgestellt werden muss. Das ist kein Prinzip ohne Ausnahmen, weil es gerade nur eine Erwartung formuliert: nur die Tatsache, daß man damit rechnen kann und daß man danach suchen darf.

2.1.4. Das letzte Prinzip - charakteristisch für den europäischen Strukturalismus, nicht aber für den Strukturalismus allgemein, nicht z.B. für den nordamerikanischen - ist das Prinzip der Neutralisierung, d.h. die Oppositionen funktionieren in der Sprache nicht immer, nicht in jedem Fall, sondern sie können an bestimmten Stellen oder in bestimmten Kontexten aufgehoben werden, neutralisiert werden, so daß ein Glied der Opposition der Funktion der gesamten Opposition entsprechen kann. So hat man z.B. wiederum im Wortschatz im Deutschen „Tag“ und „Nacht“, und „Tag“ und „Nacht“ funktionieren in dieser Opposition. „Tag“ d.h. derjenige Teil der 24 Stunden, in dem man Licht hat, „Nacht“, derjenige Teil, in dem das Licht abwesend ist. „Tag“ aber kann auch für beides funktionieren, wenn man sagt „Ich war 5 Tage in Spanien“, dann nimmt niemand an, daß ich nachts schnell nach Frankreich gegangen bin, sondern er versteht Tag und Nacht zusammen, versteht, daß diese Opposition gerade aufgehoben wird und daß ein Glied der Opposition das Ganze übernimmt. So ist es in der Phonetik, so ist es in der Grammatik, so ist es im Wortschatz. In der Grammatik ist sehr oft in unseren Sprachen der Singular z.B. neutrales oder extensives Glied, die Opposition Singular: Plural kann auch für Plural gebraucht werden, nicht aber umgekehrt. Maskulinum ist sehr oft in unseren Sprachen extensives Glied, kann auch das Femininum miteinschließen, nicht umgekehrt. Wenn in einer Prüfungsordnung z.B. „der Student“ steht, glaubt keine Studentin, daß sie dadurch nicht betroffen wird, sondern „Student“ steht für Maskulinum und Femininum. Studentinnen hingegen ist ein intensiver Terminus von Oppositionen und steht nur für Studenten weiblichen Geschlechts. Damit man sich erinnert, kann man es auch so formulieren, „die Studenten“ sind weiblich „die Studentinnen“, „die Studenten“ hingegen neutral. Dies sind also Beispiele der Möglichkeit der strukturellen Analyse, diese strukturelle Analyse bedeutet nichts anderes als festszutellen, wie eigentlich eine Sprache gemacht ist, wie sie gebaut ist.

2.2. Kann man von Strukturen des Textes sprechen? Man kann sicherlich von Strukturen des Textes sprechen, wiederum wenn es um reale Strukturen geht, um die Strukturen, die dem Inhalt des Textes entsprechen. Diese Strukturen werden entweder Strukturen des Sinnes sein oder die materiellen Strukturen, die den Sinn ausdrücken; d.h. es werden Strukturen jetzt der Bedeutung und Bezeichnung sein, aber in dieser Funktion, um einen bestimmten Sinn auszudrücken. Zuerst ein sehr einfaches Beispiel, dann ein kompliziertes. Voraussetzung ist, die sprachliche Fakten bedeuten in den Texten nicht, wie angenommen wird, etwas anderes als in der Sprache. Sie können in den Texten eine bestimmtere Bezeichnung haben, aber sie haben keine andere Bedeutung als in der Sprache, und man muss sehen, daß sie nicht etwas anderes bedeuten in einem Text, sondern was mit diesen Bedeutungen auf der Ebene des Sinnes gemacht worden ist. Dies ist auch in praktischer Hinsicht von einem großartigen Linguisten in unserem Jahrhundert gesehen worden, von einem der beiden größten Linguisten: dem Italiener Antonino Pagliaro, der, wenn auch nicht theoretisch, so doch 
praktisch stets dieses Prinzip angewandt hat, in seinen Analysen. Das Prinzip, nicht nach anderen Bedeutungen zu suchen, sondern die Bedeutung in der Sprache anzunehmen und zu sehen, was mit dieser Bedeutung dann z.B. der Heilige Franziskus gemacht hat, oder was mit dieser Bedeutung Dante gemacht hat in einem bestimmten Text ${ }^{9}$. Dies sind nebenbei gesagt die allerbesten Beispiele von Textanalysen, die mir bekannt sind, diejenigen von Antonino Pagliaro.

2.2.1. Nun, mit dieser Voraussetzung ein kleines Beispiel, ein Gedicht von Sappho. Es lautet folgendermaßen:
„,

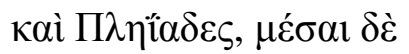

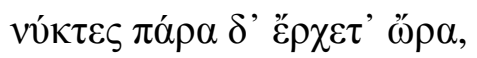
है $\gamma \omega \delta \dot{\varepsilon} \mu o ́ v \alpha \kappa \alpha \tau \varepsilon v ́ \delta \omega^{10}$.“

D.h. in der wörtlichen Übersetzung: „Der Mond schon untergegangen. Auch die Plejaden. Es ist Mitternacht. Die Zeit vergeht, und ich sitze hier allein“. So wird auch das Gedicht übersetzt, weil man m.E. gerade den Sinn des Gedichtes nicht genau versteht, und man versteht ihn deshalb nicht, weil man nicht auf die Struktur des Sinnes achtet. Diese Struktur des Sinnes ist durch zwei sprachliche Fakten gegeben. Erstens durch die Partikel des

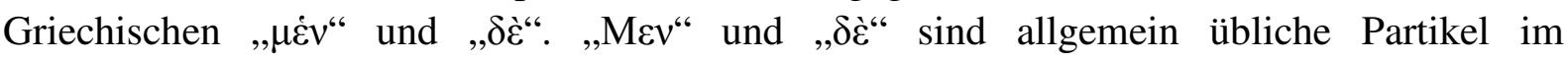
Griechischen. Sie haben die Funktion, Fakten, Ereignisse zu klassifizieren, d.h. die kategorielle Verschiedenheit zu zeigen von den besprochenen Fakten. In jedem Fall auch z.B.

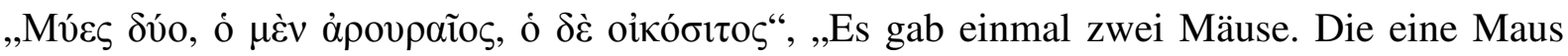

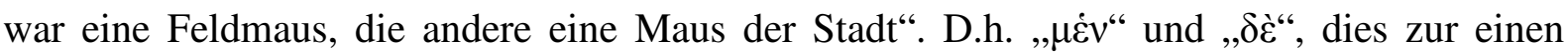
Kategorie, das anders zu einer anderen Kategorie. Nun, was steht in diesem Gedicht, so

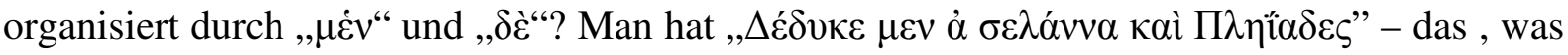

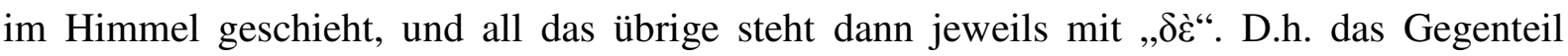
davon ist das, was auf der Erde geschieht; und auf der Erde geschieht nur folgendes: Es ist Mitternacht. Es vergeht die Zeit, und Sappho ist allein; es ist auch kein anderes Wesen auf der Welt erwähnt. Diese Einsamkeit von Sappho ist eigentlich eine komische Einsamkeit, und das Ausmaß, der Maßstab dieser Einsamkeit ist das, was im Himmel geschieht; denn das Gegenteil ist gerade nur der Himmel mit seinem Ereignissen. Un das zweite kleine

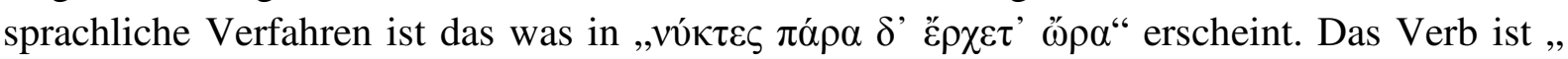
$\pi \alpha \rho \varepsilon ́ \rho \chi о \mu \alpha \iota^{\prime \prime}$ und bedeutet ,vergehen“, „vorbeigehen“. Hier erscheint es aber getrennt:

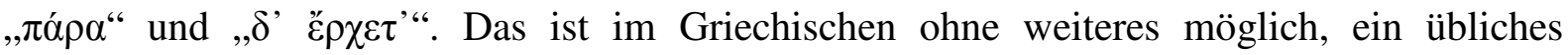
Verfahren, und man könnte sagen, sie wollte eine Silbe mehr haben. Nicht Sappho, die ein sehr großer Dichter war. Das Sie wollte sicherlich nicht, bloß eine Silbe, wenn sie das gemacht hat, dann mit einer bestimmten Absicht. Und man kann feststellen, was sich dadurch

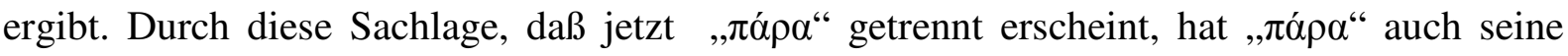

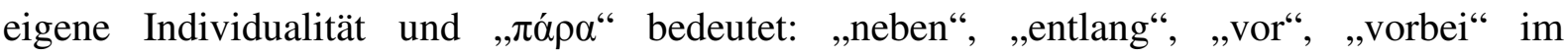

9 Vgl. z.B. „Il Cantico di Frate Sole“, Saggi di critica semantica, 1953, Firenze, Casa Editrice G. D’Anna, S.199-226; "Il canto V dello 'Infierno"', Nuovi saggi di critica semantica, 1956, Firenze, Casa Editrice G. D'Anna, S. 265-306; "Il texto della Divina Commedia e l'essegesi", Altri saggi di critica semantica, 1961, Firenze, Casa Editrice G. D’Anna, S. 183-219; "Ritmo e sintassi nel linguaggio dantesco", op.cip., S. 285295

10 Max Treu, 1955, Sappho, München, S. 211 
eigentlichen Sinne, d.h. ,an einer Sache vorbei“. Deshalb vergeht diese Zeit nicht einfach so, wie die Zeit vergeht, sondern sie geht an Sappho vorbei, sie fließt vor Sappho, und Sappho wohnt gerade dieser Tatsache bei, daß die Zeit vor ihr vorbeigeht, neben ihr vorbeigeht. Das ist also eine solche Einsamkeit, bei der gerade diese Vergehen der Zeit zu etwas Materiellen und Konkreten geworden ist.

2.2.2. Das andere Beispiel ist etwas kompliziert, aber ich werde versuchen, es kurz zu machen, weil die Zeit vergeht. Man kann das ganze Werk von Kafka als einen Text ansehen. Und man kann feststellen, was bei Kafka als Bezeichnung erscheint, d.h., was dort erzählt wird und welches die Relation jeweils mit dem Sprachlichen ist. Bei Kafka passieren Sachen wie die folgende: Man hat einen neuen Rechtsanwalt, der heißt Bucephalus. Man nimmt an, er habe nur den gleichen Namen wie das Pferd von Alexander dem Großen. Nein, es ist tatsächlich das Pferd von Alexander dem Großen, das sich dazu entschlossen hat, sich doch den Jura zu widmen, weil es in unserer Zeit doch besser ist, sich dicke Rechtsbücher zu anzusehen, als nach Indien und zu allen Gefahren zu reisen. Es geschieht folgendes: auf der Treppe erscheint ein Wesen, es ist eine Art Latten so mit Beinen aus Holz, spricht kaum, redet manchmal, es kann noch sagen, wie es heißt. Es heißt Odradek. Es ist auch nicht gefährlich, doch man sagt sich eigentlich, was mit diesem Odradek los sei. Es passiert aber auch, daß zwei Geschwister in einem Dorf einmal klopfen, und sie erfahren dann, daß ein Verbrechen in dieser Stadt geschehen ist, und daß sie - der Bruder übernimmt dann die Schuld - ihr ganzes Leben im Gefängnis bleiben müssen wegen dieses Verbrechens. Es passiert auch, daß man einen Prozess hat; man weiß nicht genau, aus welchen Gründen, aber man ist sicherlich schuldig; und am Ende wird man wie ein Hund getötet. Es passiert, daß in der Strafkolonie das Kriterium des Richters ist, der Angeklagte ist immer schuldig, grundsätzlich schon, und er kann sich auch nicht verteidigen, darf es auch nicht, weil er im Voraus schon schuldig ist.

2.2.3. Nun, diese Dinge, die passieren, haben etwas Gemeinsames, d.h. sie stehen in einer bestimmten Relation als bezeichnete Dinge: sie geschehen nicht alle nach der gleichen Norm, sondern nach verschiedenen Gesetzmäßigkeiten. Einmal kann das eine übliche Verwechslung sein, einmal kann das ein harmloses Ding wie Odradek sein, einmal kann eine harmlose Handlung ein furchtbares Verbrechen sein. Das heißt, das ist eine Welt, die aus verschiedenen Stücken gemacht ist und mit verschiedenen Gesetzmäßigkeiten. Eine Welt, die stets eine grausame Welt ist, ist nicht furchtbar im Grunde. Man weiß, womit man rechnen kann. In dieser Welt aber weiß man nicht, welches die Gesetzmäßigkeit ist, wo wir uns jetzt befinden; denn es könnte sein, daß es um das harmlose Odradek geht, oder es könnte sein, daß wir als Hunde getötet werden für eine Schuld, die wir überhaupt nicht kennen. Nun, diese furchtbare Welt steht in einer bestimmten Relation mit dem Sprachlichen und mit dem sprachlichen Verfahren, mit der Art Erzählung. Diese Welt überrascht nicht. Diese Welt ist absolut alltäglich. Die Personen, die diese verschiedenen Fakten erfahren, sind übliche, alltägliche Personen; ein Handelsvertreter, ein Angestellter - sie sind sogar anonym - sie heißer nur K., oder wenn sie einem Namen haben wie Gregor Samsa, dann ist das kein besonderer Name, es ist nur der Name eines Reisenden, eines Handelsvertreters. Und dies alles wird erzählt mit der radikalen Objektivität eines Berichtes, d.h. sogar in einer Art Verwaltungssprache, und manchmal heißt sogar die Erzählung, Bericht an eine Akademie z.B. - Und dann ganz genau die verschiedenen Fakten, oder das, was ein Vater über seine Söhne sagt. Und es wird immer wieder gesagt, das sei das Alltägliche und wird auch als Alltägliches mit dieser Art Sprache dargestellt, d.h. mit einer rein berichtenden Sprache. 
Dadurch entsteht wiederum ein Sinn. Es entsteht nämlich die Vermutung, daß diese Welt, die aus Stücken gemacht ist, diese furchtbare Welt, nicht etwa eine imaginäre Welt ist, sondern daß es die alltägliche Welt ist, d.h. unsere Welt - die Welt der kleinen Handelsvertreter, der Reisenden, der Angestellten, der anonymen Personen. D.h. diese furchtbare Welt, wo u.a. wir für Verbrechen, die wir nicht kennen, verurteilt werden können, und wo wir wie Hunde getötet werden können, das ist eigentlich unsere alltägliche Welt und keine andere. Das ist noch natürlich keine ausführliche Analyse. Ich wollte Ihnen nur zeigen, wie man Relationen auf dem Gebiet des Sinnes feststellen kann, und wie man sie interpretieren kann.

3. Ich komme leider nur sehr kurz zum dritten Teil, zum Teil Leistung und Grenzen des Strukturalismus. In Deutschland vor allem hat man immer wieder Einwände gegenüber dem Strukturalismus erhoben. Zum großen Teil aus reiner Unkenntnis, d.h. Unkenntnis der Traditionen, auch gerade der deutschen Traditionen. Derjenige, der zuerst festgestellt hat und es ausdrücklich formuliert hat, daß die Sprachen gerade gestaltet sind, nicht nur im Materiellen, sondern gerade in ihren Funktionen, ist Wilhelm von Humboldt gewesen, der kein nordamerikanischer Strukturalist war. Das hat man aber vergessen zum Teil, zum Teil hat man es auch nicht gewußt. Andererseits hat Deutschland an dieser Entwicklung kaum mitgemacht. In Deutschland har es große Vorläufer gegeben, Wegbereiter des Strukturalismus. Der Strukturalismus hat sich aber außerhalb Deutschlands aus verschiedenen Gründen, u.a. auch aus politischen Gründen entwickelt. Deshalb gab es auch ein geringes Verständnis, vor allem in Deutschland und in Italien (das ist das andere Land ohne Strukturalismus) für die strukturalistische Fragestellung. Die Einwände, die erhoben werden, sind aber meist sinnlos. Es wird z.B. angenommen, der Strukturalismus wolle die absolute Regelmäßigkeit der Sprachen. Kein vernünftiger Strukturalist hat so etwas je angenommen. Es geht nur darum festzustellen, wie die Sprache tatsächlich gebaut ist. Höchstens könnte man sagen, daß die Sprache nicht gebaut ist. Das wäre der einzige vernünftige Einwand, d.h. daß eine solche Gestaltung nicht existiert. Dem würden aber gerade die sprachliche Fakten widersprechen, denn die sprachliche Fakten sind gestaltet, erscheinen in den Sprachen als gestaltet, und die Sprachen selbst sind nichts anderes als Gestaltungen gerade der Möglichkeit der Bezeichnung von verschiedenen Gestaltungen. Auf seinem eigenen Gebiet ist deshalb, wenn man ihn richtig versteht, der Strukturalismus eigentlich nicht kritisierbar und einwandfrei. Die eigentlichen Grenzen des Strukturalismus bestehen gerade in seiner Begrenztheit; denn der Strukturalismus betrifft nur die Ebene der Einzelsprachen, so wie er bisher gemacht worden ist, und in der Einzelsprache nur eine bestimmte Sprache jeweils, eine einheitliche Sprache, wo die Strukturen festgestellt werden können; das, was ich eine funktionelle Sprache nenne. Z.B. ist es unmöglich, das Französische als Ganzes strukturalistisch zu beschreiben. Man kann nur eine bestimmte Form des Französischen jeweils beschreiben, denn man kann nicht die gleiche Opposition überall feststellen, wenn sie nicht überall da ist. Man kann nicht das Englische als Ganzes durch Oppositionen beschreiben. Wenn z.B. die Opposition „can“:,,may“ in gewissen Formen des Englischen gemacht wird und in anderen überhaupt nicht gemacht wird, weil „,may“ überhaupt nicht da ist, weil man jeweils nur „can“ sagt, auch für das, was andere Englischsprechende hingegen „may“ nennen. Das heißt das, was beschrieben werden kann, und das Gebiet, auf dem der Strukturalismus unschlagbar ist, das ist die funktionelle Sprache, eine einheitliche Sprache innerhalb einer historische Sprache; eine Sprache ohne Unterschiede in Räumen, ohne Verschiedenheit, ohne mundartliche Verschiedenheit, d.h. nur eine Mundart ohne Unterschiede, was die sozialkulturellen Schichten betrifft, d.h. ohne diastratische 
Unterschiede und ohne Unterschiede, die die Sprachstile betreffen, ohne diaphasische Unterschiede $^{11}$. Eine solche Sprache ist eine funktionelle Sprache, da sie unmittelbar in den Texten funktioniert an jedem Punkt eines Textes - jeweils nur eine bestimmte Sprache, niemand spricht deutsch, niemand spricht französisch, jeder spricht eine bestimmte Art Deutsch und in einem Text, an einem bestimmten Punkt hat man eine absolut determinierte Art Deutsch, oder eine absolut determinierte Art Französisch jeweils. Warum ist dies aber Begrenztheit? Es ist eine Begrenztheit aus folgendem Grund: Man spricht nicht nur mit sprachlichen Kompetenz, sondern man spricht zugleich mit verschiedenen anderen Kompetenzen, und wenn die Sprachwissenschaft nicht nur das sprachliche Sprechenkönnen zu beschreiben hat, sondern das Sprechen überhaupt rechtfertigen will, dann muß sie sich auf diese anderen Kompetenzen beziehen. Denn diese Kompetenzen sind stets da, und man spricht anders, nicht weil die Sprache anders ist, sondern weil diese Kompetenzen auf eine bestimmte Weise zum Sprechen beitragen. Die erste dieser Kompetenzen ist die Kenntnis der Sachen, d.h. die tatsächliche Kenntnis der Sachen in unserer Welt; entweder die allgemeine Kenntnis der Sachen oder in einer bestimmten Situation. Nur ein Beispiel dafür: Man hat immer wieder gesagt, die Körperteile hätten in verschiedenen Sprachen eine ganz bestimmte Syntax, weil man z.B. „diese Dame hat schöne Beine“ sagt, nicht aber „, diese Dame hat Beine“, weil man „dieses Kind hat blaue Augen“ sagt, nicht aber „ein Kind mit Augen“. Es stimmt nicht, daß das eine Syntax der Körperteile ist, es ist nicht einmal eine Syntax der Namen der Körperteile, sondern es ist eine allgemeine Erscheinung, die die Kenntnis der Sachen betrifft. Das heißt, das, was üblich ist, was normal angenommen wird, wird nicht gesagt. Das heißt, man sagt nicht „diese Dame hat Beine“, weil in unserer Welt alle normalen Damen Beine haben. In einer Welt, wo gerade die Damen keine Beine hätten, dann würde man es ohne weiteres sagen... - „hast du gesehen, diese Dame hat Beine!“ Wir sagen aber nur, daß diese Dame schöne Beine hat, da gerade nicht alle Damen schöne Beine haben. Das heißt also, es wird gesagt, was nicht dem im voraus Angenommen entspricht. Deshalb sagt man auch nicht, ein Fluß mit Wasser. Zu einem Fluß gehört schon das Wasser. Man sagt aber „mit klaren Wasser“ z.B., oder „mit trübem Wasser“, weil dies schon etwas über das hinaus ist, was zu einem Fluß an und für sich gehört. Und wir sagen nicht „eine Frau ohne Bart“, weil in unserer Welt normalweise die Damen keinen Bart haben oder keinen Bart tragen. In einer Welt aber, wo die Damen gerade alle Bart tragen würden und hätten, würde man ohne weiteres sagen ,eine Frau ohne Bart“, wenn man einer begegnen würde. Das heißt, man hat zunächst diese Kenntnis der Sachen. Wir wissen im Augenblick nicht einmal, in welchem Ausmaß diese Kenntnis der Sachen eigentlich zum Sprechen beiträgt und das Sprechen und die Konstitution der Texte eigentlich bestimmt. Andererseits stimmt es, daß man Strukturen in jeder funktionellen Sprache feststellen kann, und zwar saubere Strukturen. Die funktionelle Sprache aber hat den Nachteil, daß sie nicht als etwas Abgegrenztes jeweils gegeben ist, denn es gibt kaum Texte, die nur eine funktionelle Sprache realisieren; und es gibt keine Sprecher, die nur eine funktionelle Sprache sprechen können. Jeder Sprecher kann seine eigene Mundart, aber kann etwas zumindest von anderem Mundarten, zumindest passiv und bis zu einem gewissen Punkt kann er auch diese anderen Mundarten gebrauchen: Er spricht schwäbisch, aber er weiß, daß man in Berlin $g$ als $j$ ausspricht, zumindest so viel weiß er, und er weiß, daß er in Berlin ,jab“ usw. sagen würde.

11 Vgl. hierzu Eugenio Coseriu, 2001, Vers l'étude des structures lexicales [1966], in L'homme et son langage, Louvain, Peeters, S. 239ff. ; Eugenio Coseriu, 1998, Le double problème des unités « dia-s », Les Cahiers $\delta i \alpha$. Etudes sur la diachronie et la variation linguistique, I, S. 9-16 
Jeder Sprecher spricht zwar ein bestimmtes Niveau, ein bestimmtes Sprachniveau; er kennt aber zum Teil auch andere Sprachniveaus und kann sie nachahmen, kann sich sogar dabei irren, gerade indem er andere Sprachniveaus nachahmt, indem er annimmt, daß es so beim anderen Sprachniveau sei. Diese Nachahmung, auch gerade die irrtümliche zeigt doch eine bestimmte Kenntnis, und jeder Sprecher spricht per definitionem verschiedene Sprachstile, d.h. er kann familiär sprechen z.B., er kann auf eine feierliche Weise sprechen und anderes mehr. Eine Beschreibung jeder dieser funktionellen Sprachen getrennt, d.h. je eine Beschreibung, auch wenn sie sicherlich sehr kompliziert wäre, wäre zwar möglich dies würde aber das eigentliche Problem nicht lösen. Das eigentliche Problem ist nicht, die Gesamtheit der funktionellen Sprachen in einer historischen Sprache zu beschreiben; das Problem ist zu erfahren, was ein Sprecher, zumindest ein einziger Sprecher, als „Sprechen-können“ tatsächlich besitzt, in welchem Ausmaß z.B. er zwar bei einer bestimmten funktionellen Sprache verankert ist, aber zugleich verschiedene andere teilweise kennt. Z.B. was wissen alle Deutschen von den übrigen Mundarten, von den Mundarten, die nicht jeweils ihre eigene Mundart sind? Man weiß etwas über das Kölnische, etwas über das Bairische und man kann es bis zu einem gewissen Punkt auch gebrauchen. Deshalb kann eine strukturelle Sprachbeschreibung, die zwar einer funktionellen Sprache genau entspricht, nicht dem eigentlichen „Sprechkönnen“ eines Sprechers entsprechen, weil dieses „Sprechkönnen“ schon heterogen ist und verschiedenes aus verschiedenen funktionellen Sprachen enthält. Ich muß mich hier auf diese beiden Grenzen des Strukturalismus beschränken, d.h. einerseits die Kenntnis der Sachen: wir haben keine Linguistik, die eine skeuologische Linguistik (von

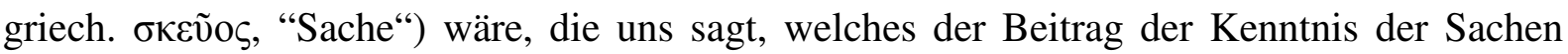
zum Sprechen ist ${ }^{12}$, und andererseits: wir haben keine poly-funktionelle Linguistik, die uns sagt, inwiefern ein Sprecher einer Sprache gerade über diese Pluralität von Einzelsprachen innerhalb seiner eigenen historischen Sprache verfügt. Dies müsste gerade als Überwindung des Strukturalismus verstanden werden, d.h. in diesem Sinne kann man zu einer integralen Linguistik kommen, die die verschiedenen anderen Faktoren, die für das Sprechen bestimmend sind, berücksichtig. Bei einer solchen Linguistik aber würden die Errungenschaften des Strukturalismus nicht verschwinden, sondern im Gegenteil. Der Strukturalismus ist für sein Gebiet, für die funktionelle Sprache, das Geeignete, d.h. in dieser integralen Linguistik würde der Strukturalismus immer noch der Kern bleiben für das, was die Beschreibung der einzelnen Sprache als funktioneller Sprache angeht.

12 Über die skeuologische Linguistik, vgl. Eugenio Coseriu, 1981, „La socio - y la etnolingüística. Sus fundamentos y sus tareas“, Anuario de Letras, XIX, México, S. 10-11; Eugenio Coseriu, 1992, Strukturelle und kognitive Semantik, Vorlessung WS 1989/1990, Nachschrift von Ulrike Maier und Heinrich Weber, Tübingen, S. 60Af.; Eugenio Coseriu, 2000, „Structural semantics and ,cognitive“ semantics“, Logos and Language, I:1, S. 32f. 